\title{
Conducting Polymer Scaffolds Based on Poly(3,4- ethylenedioxythiophene) and Xanthan Gum for Live-Cell Monitoring
}

Isabel del Agua, ${ }^{\dagger, \ddagger, \S}$ Sara Marina, ${ }^{\ddagger}$ Charalampos Pitsalidis, ${ }^{\ddagger, \|}$ Daniele Mantione, ${ }^{\ddagger, \perp}$ Magali Ferro, ${ }^{\ddagger}$ Donata Iandolo, ${ }^{\ddagger}, \|$ Ana Sanchez-Sanchez, ${ }^{\ddagger, \#}$ George G. Malliaras, ${ }^{\ddagger}, \#$ Róisín M. Owens, ${ }^{*}$, , and David Mecerreyes* ${ }^{\dagger}$, II

${ }^{\dagger}$ POLYMAT University of the Basque Country UPV/EHU, Joxe Mari Korta Center, Avda. Tolosa 72, 20018 Donostia-san Sebastian, Spain

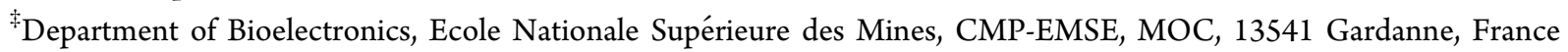

${ }^{\S}$ Panaxium SAS, 67 Cours Mirabeau, 13100 Aix-en-Provence, France

"Department of Chemical Engineering and Biotechnology, Philippa Fawcett Drive, Cambridge CB3 OAS, U.K.

${ }^{\perp}$ Laboratoire de Chimie des Polymères Organiques, Université Bordeaux/CNRS/INP, Allée Geoffroy Saint Hilaire, Bâtiment B8, 33615 Pessac Cedex, France

\# Department of Engineering, Electrical Engineering Division, University of Cambridge, 9 JJ Thomson Avenue, Cambridge CB3 0FA, U.K.

II Ikerbasque, Basque Foundation for Science, E-48011 Bilbao, Spain

Supporting Information

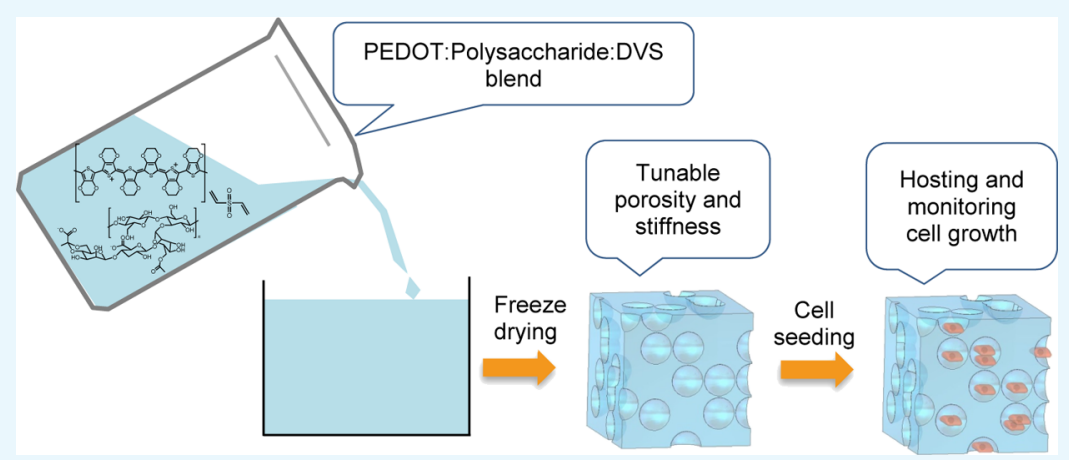

ABSTRACT: Conducting polymer scaffolds can promote cell growth by electrical stimulation, which is advantageous for some specific type of cells such as neurons, muscle, or cardiac cells. As an additional feature, the measure of their impedance has been demonstrated as a tool to monitor cell growth within the scaffold. In this work, we present innovative conducting polymer porous scaffolds based on poly(3,4-ethylenedioxythiophene) (PEDOT):xanthan gum instead of the well-known PEDOT:polystyrene sulfonate scaffolds. These novel scaffolds combine the conductivity of PEDOT and the mechanical support and biocompatibility provided by a polysaccharide, xanthan gum. For this purpose, first, the oxidative chemical polymerization of 3,4-ethylenedioxythiophene was carried out in the presence of polysaccharides leading to stable PEDOT:xanthan gum aqueous dispersions. Then, by a simple freeze-drying process, porous scaffolds were prepared from these dispersions. Our results indicated that the porosity of the scaffolds and mechanical properties are tuned by the solid content and formulation of the initial PEDOT:polysaccharide dispersion. Scaffolds showed interconnected pore structure with tunable sizes ranging between 10 and $150 \mu \mathrm{m}$ and Young's moduli between 10 and $45 \mathrm{kPa}$. These scaffolds successfully support threedimensional cell cultures of MDCK II eGFP and MDCK II LifeAct epithelial cells, achieving good cell attachment with very high degree of pore coverage. Interestingly, by measuring the impedance of the synthesized PEDOT scaffolds, the growth of the cells could be monitored.

\section{INTRODUCTION}

Three-dimensional (3D) cell culture in artificial environments finds numerous applications. It is currently used for cancer research, drug discovery, and regenerative medicine among
Received: March 12, 2018

Accepted: June 20, 2018

Published: July 6, 2018 


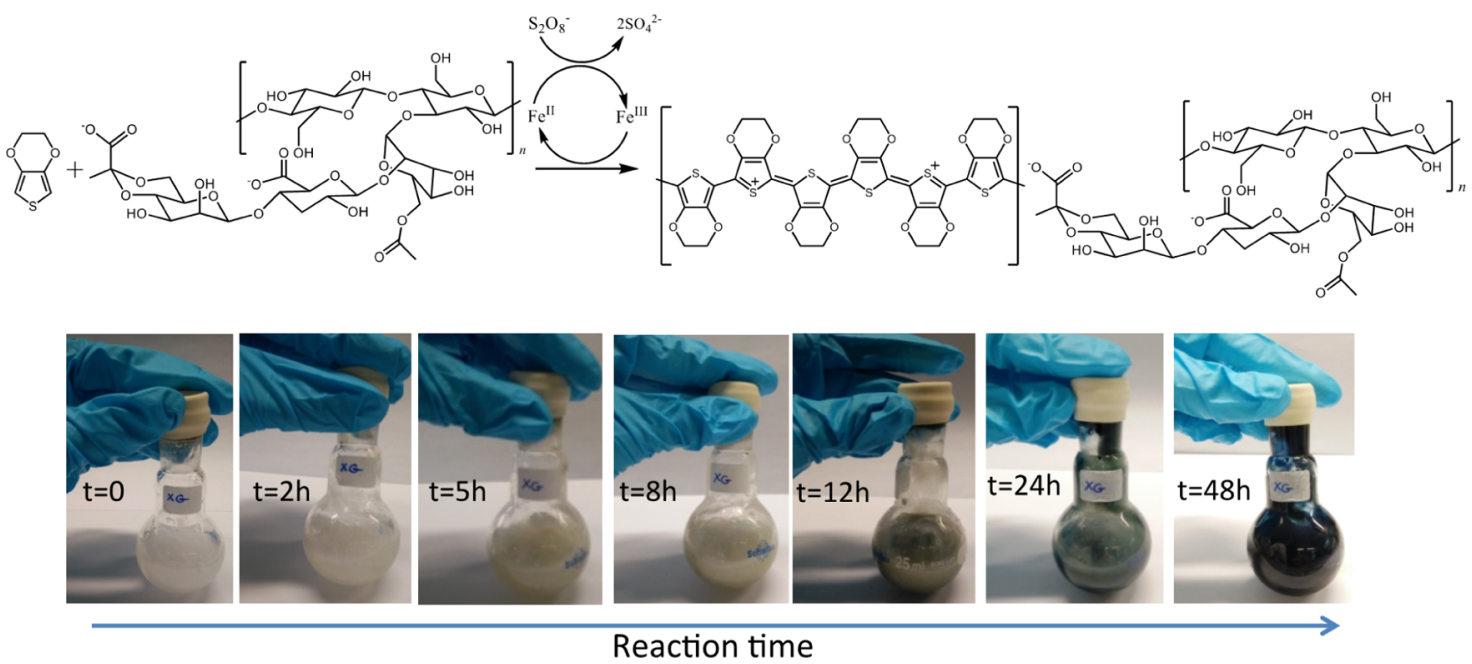

Figure 1. PEDOT:xanthan gum synthetic scheme and pictures of the reaction flask at different times.

many other research areas. To culture cells in vitro, 3D structures that support cell attachment and guide cells for tissue development are needed. ${ }^{1}$ These structures are normally made of polymeric materials, that is, polymers which allow designing scaffolds with certain structural and mechanical properties. $^{2}$ Among these scaffold properties, their porosity and stiffness are of crucial importance. Their void structure is a key factor as interconnected pores create pore networks, which aid diffusion of nutrients and oxygen and removal of waste, ${ }^{3}$ and facilitate scaffold colonization. ${ }^{4}$ The mechanical behavior of the scaffold should mimic the extracellular matrix (ECM), ${ }^{2}$ providing an environment as similar as possible to the cell natural environment.

As a particular type of scaffold, conducting polymer scaffolds are currently interesting for their additional advantage of promoting cell growth by electrical stimulation. Conducting polymers used in tissue engineering are polypyrrole, ${ }^{5,6}$ polyaniline, ${ }^{7,8}$ polythiophenes, ${ }^{9,10}$ and poly(3,4-ethylenedioxythiophene) (PEDOT). ${ }^{11-13}$ They have been used to enhance bone, muscle, and nerve tissue regeneration. ${ }^{6}$ Moreover, they are also used in drug delivery, with drugs bound in the scaffolds and released through an electrical signal. ${ }^{14}$ Conducting scaffolds can be synthesized by many techniques and, in particular, PEDOT scaffolds have been prepared ${ }^{15}$ by chemical, vapor-phase polymerization ${ }^{16}$ of and by electrochemical polymerization $^{17,18}$ of 3,4-ethylenedioxythiophene (EDOT) monomer around a previously synthesized scaffold. Another alternative is to use the freeze-drying method to fabricate porous PEDOT:polystyrene sulfonate (PSS) 3D scaffolds. ${ }^{19,20}$ This method allows adding different additives to improve mechanical robustness and stability of the scaffold or even proteins such as collagen to improve cell growth prior to the freeze-drying step. As a new application recently, a livecell monitoring platform was demonstrated by using a conducting polymer scaffold based on PEDOT. ${ }^{20}$ The inclusion of cells within the porous scaffold affects the impedance of the electrically conducting polymer network, and thus, it can be used as an in situ tool to monitor the cell growth.

For their successful interaction with cells, the polymeric scaffolds should act as an artificial ECM. ECM in the human body is composed of proteins and polysaccharides. ${ }^{21}$ For this reason, it is ideal that these biopolymers are used in the scaffold design. ${ }^{22,23}$ In fact, polysaccharides form hydrogels in water, reducing the stiffness of the scaffolds to values similar to that in biological tissues. Among the employed polysaccharides, xanthan gum presents special interest because of its processability and biocompatibility. ${ }^{24,25}$ For instance, xanthan gum scaffolds have been used to improve osteoblast growth ${ }^{26}$ and fibroblast proliferation. ${ }^{5}$ We present in this work a new synthetic route to elaborate conducting PEDOT:xanthan gum scaffolds that can be used for cell growth monitoring. As a result of the polysaccharide content, these scaffolds better mimic the ECM than PEDOT:PSS scaffolds. The scaffold preparation consists of two steps. First, an aqueous dispersion of PEDOT:polysaccharide is synthesized by oxidative polymerization of EDOT in the presence of xanthan gum as the stabilizer. Second, a conductive porous scaffold is obtained by freeze-drying the PEDOT:xanthan gum dispersions. As an application, the conducting $3 \mathrm{D}$ scaffolds were used for hosting and monitoring the growth of MDCK cells.

\section{RESULTS AND DISCUSSION}

\subsection{Synthesis and Characterization of PEDOT:Xan-} than Gum Aqueous Dispersions. Recently, the synthetic route toward PEDOT:polysaccharide dispersions has been reported and these materials combine the conductivity of PEDOT and the biocompatibility of polysaccharides such as dextran sulfate, ${ }^{27}$ sialic acid, ${ }^{28}$ pectin, ${ }^{29}$ hyaluronic acid, ${ }^{30}$ chondroitin sulfate, ${ }^{31}$ or guar gum. ${ }^{32}$ In this work, a similar method was applied to synthesize PEDOT:xanthan gum aqueous dispersions. The polymerization reaction can be found in Figure 1. EDOT monomer was polymerized in the presence of the polysaccharide xanthan gum by oxidative polymerization in water at room temperature using ammonium persulfate $\left(\left(\mathrm{NH}_{4}\right)_{2} \mathrm{~S}_{2} \mathrm{O}_{8}\right)$ as an oxidant in the presence of iron(III) sulfate $\left(\mathrm{Fe}_{2}\left(\mathrm{SO}_{4}\right)_{3}\right)$ as the catalyst. As the polymerization of EDOT is taking place, the dispersion first turns more yellowish (from 2 to $8 \mathrm{~h}, 0.2$ monomer conversion) to more grayish at $12-24 \mathrm{~h}(0.4$ monomer conversion $)$ to then acquire the bright blue color characteristic of PEDOT chains, which starts to be visible after $48 \mathrm{~h}$ of reaction time. The reaction takes place slowly and the initial very viscous solution becomes less viscous, which was also observed in previous PEDOT:polysaccharide systems, and it is associated with the breaking of the strong intramolecular hydrogen bonding of the poly- 

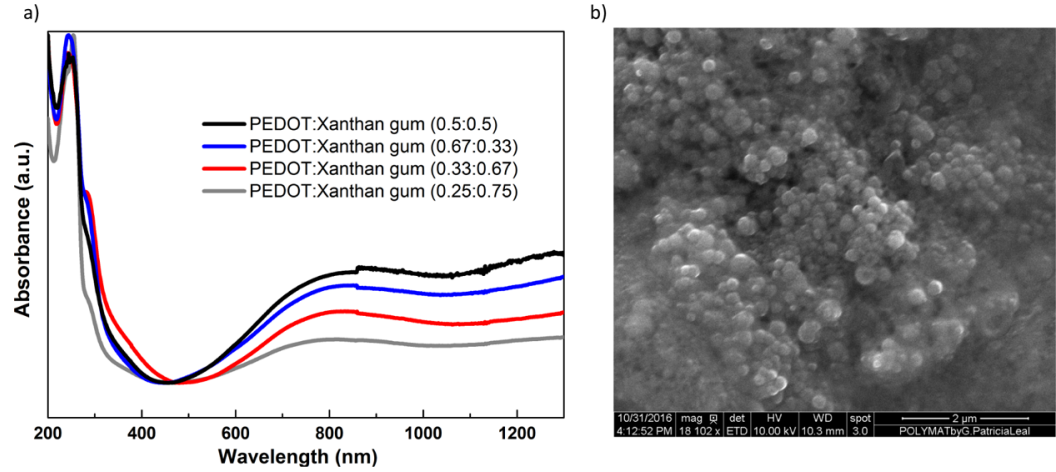

Figure 2. PEDOT:xanthan gum dispersion characterization. (a) UV spectra of PEDOT:xanthan gum dispersions at different ratios and (b) representative SEM picture of a drop-casted PEDOT:xanthan gum dispersion $(0.5: 0.5)$.

saccharides by the PEDOT chains. The monomer consumption was followed by high-pressure liquid chromatography (HPLC), confirming that the full conversion was reached after 7 days.

The chemical oxidation polymerization method to obtain PEDOT:polysaccharide aqueous dispersions is very versatile, allowing the preparation of PEDOT:xanthan gum in different ratios. Thus, we synthesized four different dispersions with the PEDOT:xanthan gum wt ratios 0.25:0.75, 0.33:0.67, 0.5:0.5, and $0.67: 0.33$. These dispersions were characterized by UVvis-NIR spectroscopy as shown in Figure 2a. In the four dispersions studied, two characteristic PEDOT absorption bands are observed. The broad absorption band of PEDOT $\pi-\pi^{*}$ centered at $\sim 800 \mathrm{~nm}^{33}$ and a bipolaron band at higher wavelengths centered at $1200 \mathrm{~nm}$. These two absorption bands indicate the presence of doped PEDOT chains as cation and dication forms ${ }^{34}$ and they are related to the conductivity of the material, confirming the conducting nature of the PEDOT chains in these PEDOT:xanthan gum dispersions. Although the absorbance of both bands is visible in the four dispersions studied, it is higher in the case of PEDOT:xanthan gum (0.5:0.5). This indicates the higher presence of doped chains in this PEDOT dispersion, which is a sign of high conductivity.

PEDOT:xanthan gum films can be easily prepared by casting these dispersions. One typical picture of these drop-casted films is shown in Figure $2 \mathrm{~b}$. As observed in other PEDOT:polysaccharide systems, ${ }^{31,32}$ the dispersions are formed by spherical PEDOT particles covered by xanthan gum of sizes ranging between 100 and $200 \mathrm{~nm}$. PEDOT spherical particles are formed as a result of PEDOT chains being surrounded by the stabilizer. When the dispersions are drop-casted, the spherical particles agglomerate together in the form of a film as shown in the scanning electron microscopy (SEM) image. This allows the intimate mixing of both PEDOT and xanthan gum. The electrical conductivity of the films of PEDOT:xanthan gum $(0.5: 0.5,0.67: 0.33)$ was also characterized by a four-point probe showing values of $10^{-2} \mathrm{~S} \mathrm{~cm}^{-1}$.

2.2. Preparation and Characterization of PEDOT:Xanthan Gum Scaffolds by the Freeze-Drying Method. In the second step, porous PEDOT:xanthan gum scaffolds were prepared by freeze-drying technique as illustrated in Figure 3. In our case, we used divinyl sulfone (DVS) as an additive to keep the integrity of the scaffolds. The choice of DVS is due to recent studies, ${ }^{35,36}$ where DVS has proven to be an effective cross-linker for PEDOT films, showing excellent biocompatibility and neuroregeneration as compared to the conventional cross-linkers. It is well known that different parameters such as

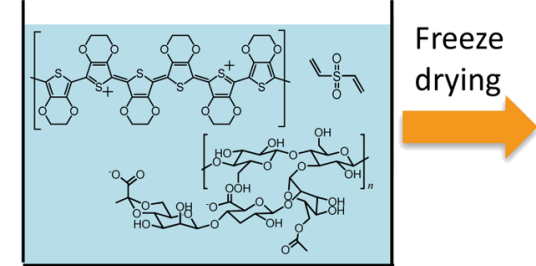

PEDOT:Xanthan Gum:DVS dispersion

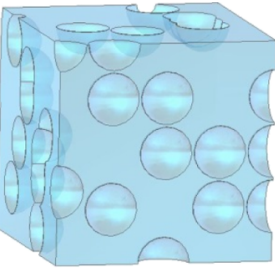

Porous Scaffold
Figure 3. Illustration of the preparation of PEDOT:xanthan gum scaffolds.

the polymer concentration of the initial dispersion, the freezedrying conditions, or the PEDOT:xanthan gum ratio may affect the morphology of the scaffold.

To study the effect of the polymer concentration, PEDOT:xanthan gum (0.5:0.5) dispersions were prepared at three different solid contents $(1,2$, and $3 \mathrm{wt} \%$ in water). After the freeze-drying step, SEM images of these different scaffolds were taken (Figure 4). In Figure $4 a$, the 1 wt $\%$ scaffold shows a porous structure based on a network of fibers. In Figure $4 b$, the $2 \%$ solid content scaffold shows pores with high polydispersity and pore size around $ø 50-150 \mu \mathrm{m}$. In Figure $4 \mathrm{c}$, the $3 \%$ solid content scaffold shows smaller pores with less polydispersity and pore size around $\oslash 10-30 \mu \mathrm{m}$. This experiment illustrates how the porosity of these scaffolds can be varied, which is an advantage of the freeze-drying method. According to the type of cell that will be studied, the porosity can be selected by varying the initial solid content of the synthesized PEDOT:xanthan gum dispersion. ${ }^{37}$ The highly interconnected porous structure of the scaffolds is also clearly visible in these SEM images. This represents a second advantage of this method, as the interconnection of pores facilitates mass transport and cell migration within the scaffold.

Second, the effect of the PEDOT:xanthan gum ratio on the structure and mechanical properties of the scaffold was investigated. In this case, the PEDOT scaffolds from aqueous dispersion having $2 \mathrm{wt} \%$ solid content were chosen because of the intermediate pore size $(50-150 \mu \mathrm{m} ø)$ of the generated scaffolds, a pore size that facilitates cell nutrient percolation, cell attachment, and colonization. ${ }^{38,39}$ Figure 5 shows the SEM images of PEDOT:xanthan gum scaffolds obtained at different ratios $(0.25: 0.75,0.33: 0.67,0.5: 0.5$, and 0.67:0.33). By comparing the SEM pictures, it is observed that at high content of PEDOT (Figure 5a), scaffolds with small pores and thick walls connecting these pores are obtained. On the other 

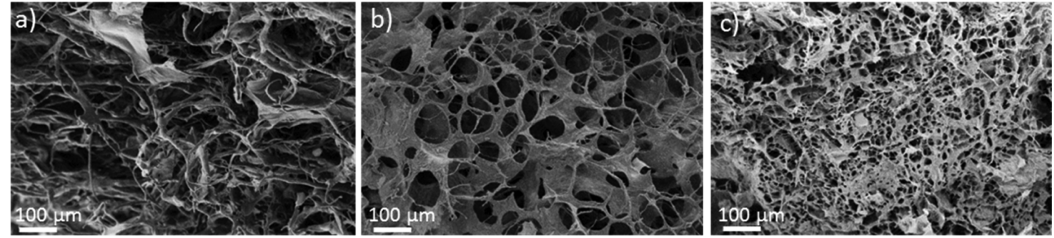

Figure 4. SEM porosity characterization of PEDOT:xanthan gum scaffolds. (a) PEDOT:xanthan gum (0.5:0.5) 1\% solid content, (b) PEDOT:xanthan gum (0.5:0.5) 2\% solid content, and (c) PEDOT:xanthan gum (0.5:0.5) 3\% solid content.

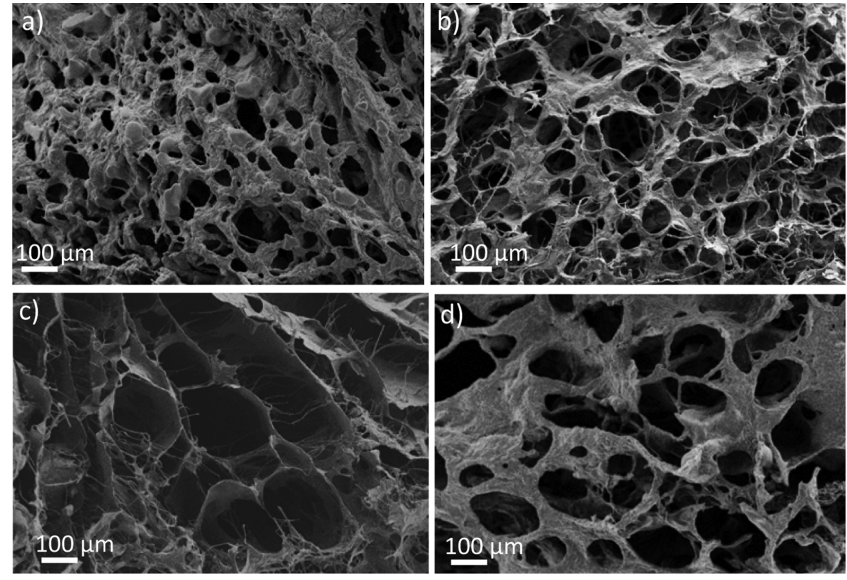

Figure 5. SEM images of PEDOT:xanthan gum scaffolds at different PEDOT:xanthan gum weight ratios. (a) PEDOT:xanthan gum (0.67:0.33), (b) PEDOT:xanthan gum (0.5:0.5), (c) PEDOT:xanthan gum (0.33:0.67), and (d) PEDOT:xanthan gum (0.25:0.75).

hand, at high xanthan gum content (Figure $5 \mathrm{~d}$ ), the scaffold shows the biggest pores and the highest porosity.

Significant differences among scaffolds were observed by studying the mechanical properties of the scaffolds by compression test. The compression test was performed both on dry and wet scaffolds after $8 \mathrm{~h}$ in phosphate-buffered saline (PBS) buffer showing values between 1 and $100 \mathrm{kPa}$ (Figure 6). As a general trend, the higher the PEDOT content, the

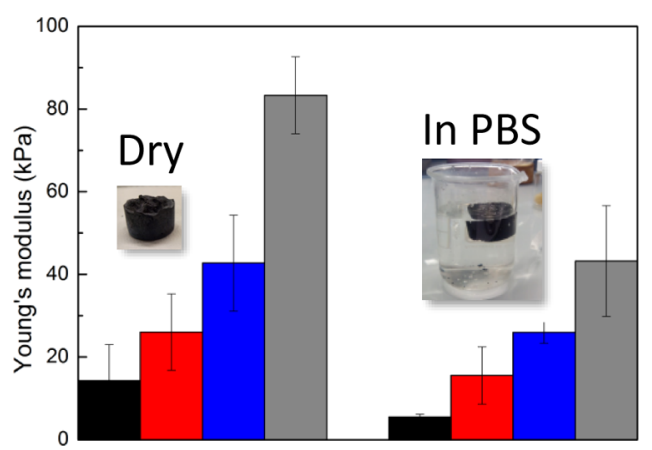

Figure 6. Young's modulus of PEDOT:xanthan gum scaffolds measured in dry and in PBS solution at different ratios (0.25:0.75) black, $(0.33: 0.67)$ red, $(0.5: 0.5)$ blue, and (0.67:0.33) gray.

higher the Young's modulus of the scaffolds. This is somehow expected because xanthan gum is a soft polymer making the scaffold more flexible overall. On the contrary, PEDOT chains are stiff, and the higher the content of PEDOT in the scaffold, the higher the Young's modulus of the scaffold. Once the scaffolds are in PBS solution, the scaffolds hydrate and swell, becoming soft but keeping their overall structure. We observed a reduction in the Young's moduli in all cases to approximately half the value than those in dry conditions. It is worth to note that similar values were reported for PEDOT:PSS scaffolds (73 $\mathrm{kPa}$ dry and $42 \mathrm{kPa}$ in PBS). ${ }^{40}$ The Young's modulus values of these scaffolds are all $<100 \mathrm{kPa}$, which corresponds with the values of our body tissues, where Young's modulus is in the range $1-100 \mathrm{kPa}$ depending on the tissue. ${ }^{41}$ The polysaccharide content of these scaffolds helps to reduce the mismatch of rigidity between a synthetic scaffold and the ECM. In conclusion, this is another way in which we can show that this synthetic way to obtain PEDOT scaffolds is very versatile as not only porosity but also the stiffness of the scaffolds can be varied by varying the initial content of PEDOT and polysaccharide.

2.3. PEDOT:Xanthan Gum Scaffolds for Hosting and Monitoring 3D Cell Cultures. As recently demonstrated, conducting scaffolds can be used as platforms for cell sensing. The cell growth and colonization of the scaffold brings changes in its electrochemical impedance. ${ }^{42}$ This change can be used to track cell growth. Prior to these experiments, the cell growth and stability of these scaffolds in cell media was assessed. Only one type of scaffold was selected to undergo the cell study. The scaffold PEDOT:xanthan gum (0.5:0.5) 2 wt \% was selected because it showed a good compromise between mechanical stability and porosity. For that MDCK II eGFP cells were seeded on a sterilized scaffold (Figure 7) with a density of 0.22
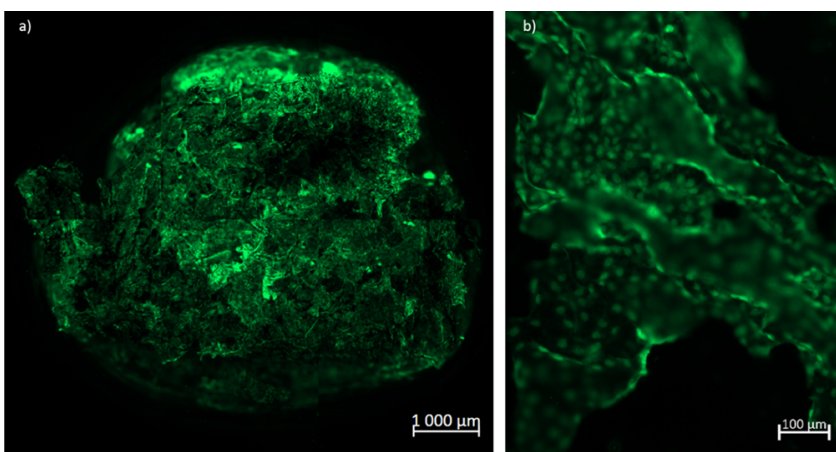

Figure 7. MDCK II eGFP cell growth on PEDOT:xanthan gum (0.5:0.5) $2 \%$ solid content scaffold 14 days after culture, $37^{\circ} \mathrm{C}, 5 \%$ $\mathrm{CO}_{2}$. (a) Confocal microscopy image of the entire scaffold 14 days after cell seeding and (b) zoomed image of the scaffold 14 days after cell seeding.

$\pm 0.05 \mathrm{~g} \cdot \mathrm{cm}^{-3}$ and swelling behavior included in Figure S1 (Supporting Information), reaching equilibrium within $24 \mathrm{~h}$. MDCK II eGFP cells present a constitutive expression of GFP protein without any requirement of media additives for the expression of green fluorescent protein over time. Therefore, we were able to dynamically evaluate cell growth into the scaffold by fluorescent analysis using conventional media. After 7 days of culture at $37^{\circ} \mathrm{C}, 5 \% \mathrm{CO}_{2}$, scaffolds maintained their 

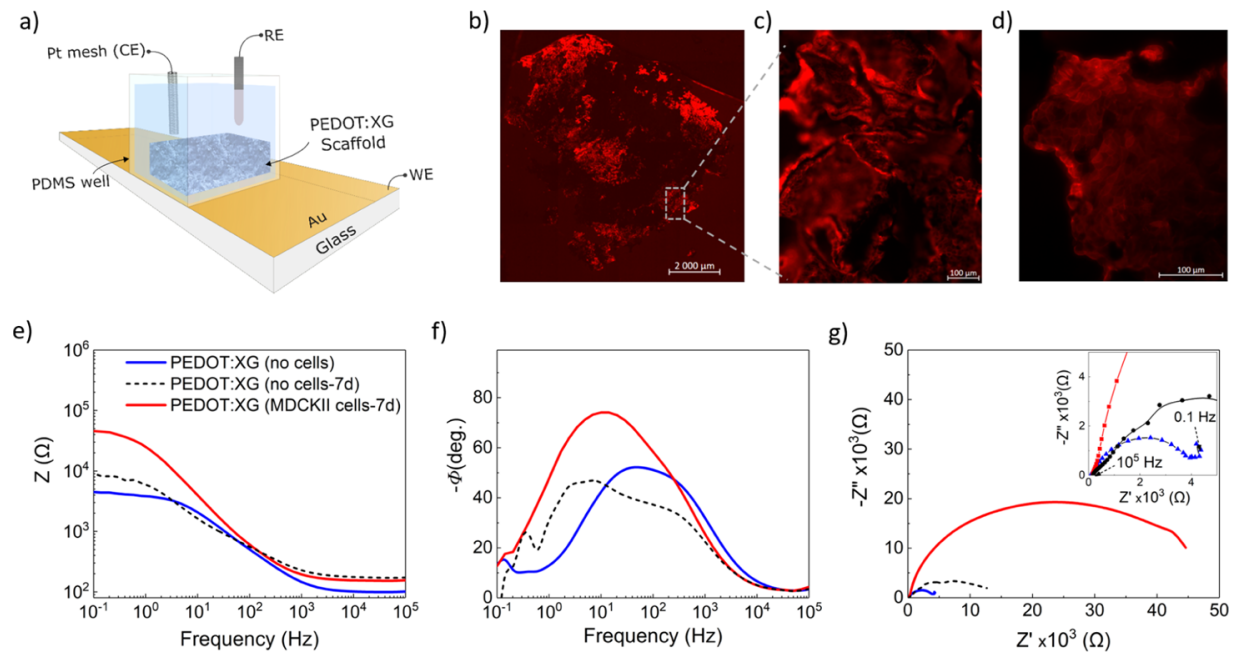

Figure 8. Cell growth on PEDOT:xanthan gum (0.5:0.5) $2 \%$ solid content scaffolds and impedance measurements. (a) Device configuration of the scaffold-based electrode. (b) Confocal microscopy image of the entire scaffold 7 days after cell seeding with fluorescent MDCK II LifeAct cells and $(c, d)$ zoomed images of the scaffold 7 days after cell seeding. Impedance spectra showing the (e) complex impedance data, (f) the phase angle vs frequency, and the $(\mathrm{g})$ Nyquist plots of the scaffolds before and after cell culture for 7 days. The dashed lines represent the spectra of a PEDOT:xanthan gum scaffold after 7 days of incubation $\left(37^{\circ} \mathrm{C}, 5 \% \mathrm{CO}_{2}\right)$ in culture media without cells.

structural integrity and we observed satisfactory coverage. We were able to observe good cell attachment and high degree of pore coverage presumed to be due to the roughness of the structure. Through the interconnection of pores within the structure, cells were able to migrate throughout almost the whole scaffold.

Knowing that the PEDOT:xanthan gum scaffolds can efficiently facilitate cell culture, we investigated the electrical properties by means of electrochemical impedance spectroscopy (EIS) and studied their evolution during cell growth as an in situ tool to monitor cell growth. For this experiment, we used MDCK II LifeAct, which has been previously used as an MDCK II cell line growing in a PEDOT:PSS scaffold, ${ }^{20}$ demonstrating their ability to form an electrical barrier and the same initial adhesion and proliferation rate as the MDCK II eGFP cells. Cells were seeded on a sterilized scaffold PEDOT:xanthan gum (0.5:0.5) 2 wt \% and an electrodebased device was fabricated as shown in Figure 8a. Specifically, gold electrodes were patterned on top of glass substrates, while a polydimethylsiloxane (PDMS) "well" was used to confine the scaffolds inside the electrode area. The $\mathrm{Ag} / \mathrm{AgCl} / \mathrm{KCl}$ (3 M) reference electrode and platinum $(\mathrm{Pt})$ mesh counter electrode were immobilized inside the wells. Figure $8 b-d$ shows the cell growth on the PEDOT:xanthan gum scaffold after 7 days of cell culture. The impedance data (presented as Bode plot) of Figure $8 \mathrm{e}$ revealed a significant increase in the impedance value of almost an order of magnitude (from $|Z| \approx 4.5 \mathrm{k} \Omega$ to $\left|Z_{\text {cell }}\right| \approx$ $45 \mathrm{k} \Omega$ ) in the case of cells containing scaffolds, in the low frequency regime $(0.1-100 \mathrm{~Hz})$. Although both sets of phase data (Figure 8f) (with and without cells) appeared to generate two time constants, an increase of $\sim 22^{\circ}$ (from $\varphi \approx 52^{\circ}$ to $\varphi_{\text {cell }}$ $\approx 74^{\circ}$ ) along with a shift toward lower frequencies is apparent. Such changes indicate the introduction of additional capacitance elements in the circuit, which corresponds to the growth of insulating cell layers in the pores of the scaffold. ${ }^{43} \mathrm{~A}$ less pronounced change can be observed for mid to high frequencies, with a slight increase in the impedance values most likely associated with the presence of cells contributing to the increase of the Ohmic resistance at the working electrodeelectrolyte interface. The Nyquist plot of Figure $8 \mathrm{~g}$ further confirmed the effects of cell growth inside the scaffold. Specifically, an increase in the radius of the semicircle in the case of control experiments was achieved by incubating (at 37 ${ }^{\circ} \mathrm{C}$ ) an identical PEDOT:xanthan gum scaffold in cell media for 7 days (dashed line in Figure $8 \mathrm{e}-\mathrm{g}$ ). A slight increase in the magnitude of impedance can be observed, which is accompanied by a small change in the phase angle. This can be likely attributed to gradual changes in the chemical composition of PEDOT scaffolds or to the presence of cell culture medium. ${ }^{11}$ Nevertheless, this cannot override the effects of cell growth in the scaffold-based electrode. It should be noted that because of the fluctuations in the pore homogeneity, the effect of cell growth on the measured impedance values may vary. The abovementioned findings are consistent with previous EIS studies of $3 \mathrm{D}$ cell cultures in PEDOT-based conducting scaffolds. ${ }^{20}$

\section{CONCLUSIONS}

In this article, a new synthetic strategy for preparing conducting PEDOT:xanthan gum scaffolds with the ability to host and monitor cells is presented. Soft and flexible conducting scaffolds were obtained by first synthesizing and second freeze-drying PEDOT:xanthan gum dispersions. The mechanical properties and porosity of the conducting scaffolds can be tuned with the concentration of the dispersion and the ratio in between the conducting polymer and polysaccharide. Scaffold pore size ranges between 10 and $150 \mu \mathrm{m}$ and Young's moduli range between 10 and $45 \mathrm{kPa}$, giving the possibility to optimize pore size and stiffness according to the target cell culture. As a potential application, these scaffolds show the ability to monitor cell growth by measuring the impedance of the scaffolds at different times. In future works in our laboratory, other polysaccharides (or mixtures of polysaccharides) will be included in these scaffolds using this synthetic methodology.

\section{EXPERIMENTAL SECTION}

4.1. Synthesis of PEDOT:Xanthan Gum Dispersions. PEDOT:xanthan gum dispersions with 1, 2, and $3 \%$ solid 
content and at different PEDOT:xanthan gum ratios were synthesized by oxidative polymerization according to the following procedure. In a $25 \mathrm{~mL}$ round-bottom flask containing $19.6 \mathrm{~mL}$ of Milli-Q water, $0.3,0.27,0.2$, or $0.13 \mathrm{~g}$ of xanthan gum (Acros) was dissolved and purged with $\mathrm{N}_{2}$. Afterward, $0.1,0.13,0.2$, or $0.27 \mathrm{~g}$ of EDOT monomer (Acros), together with the 1.5 equiv of ammonium persulfate $\left(\left(\mathrm{NH}_{4}\right)_{2} \mathrm{~S}_{2} \mathrm{O}_{8}\right.$, Sigma $)$ and a catalytic amount $(5 \mathrm{mg})$ of iron(III) sulfate $\left(\mathrm{Fe}_{2}\left(\mathrm{SO}_{4}\right)_{3}\right.$ Sigma) was added, respectively. The reaction solution was kept at $0{ }^{\circ} \mathrm{C}$ until completion (144 h). The dispersions were purified by dialysis with Milli-Q water and a regenerated cellulose dialysis membrane $(12-14 \mathrm{kD})$ from SpectrumLabs. Finally, dispersions containing different PEDOT:xanthan gum ratios were obtained by varying the initial amount of grams $(0.25: 0.75,0.33: 0.67,0.5: 0.5$, and $0.67: 0.33)$.

4.1.1. Dispersion Characterization. UV-vis-NIR measurements were taken with UV/vis/NIR spectrometer PerkinElmer Lambda 950. Employing quartz cuvettes, a $2 \mathrm{v} / \mathrm{v} \%$ solution in Milli-Q water of each PEDOT:xanthan gum dispersion was analyzed. Spectra were normalized. The kinetics of the polymerization reaction of PEDOT:xanthan gum (0.5:0.5) 2 wt \% were monitored during 7 days by HPLC using a HewlettPackard HPLC series 1100 equipment with a Lichrosphere $100 \mathrm{CN}(5 \mu \mathrm{m})$ column supplied by Agilent Technologies. The eluent is water/acetonitrile (ACN) 1:1 $0.7 \mathrm{~mL} \mathrm{~min}^{-1}$ at room temperature (r.t.). Reaction samples were taken every 30 min according to the following procedure: $10 \mu \mathrm{L}$ of the reaction sample mixture was added to $5 \mathrm{~mL}$ of $\mathrm{ACN}$ containing $1 \mu \mathrm{L}$ of benzyl alcohol as the internal standard and $20 \mu \mathrm{L}$ of this solution is injected into the HPLC equipment. EDOT concentration is calculated by plotting the area of each EDOT peak (5.3 min retention time) in a calibration line. The viscosity of the reaction solution was measured with an SV-10 Vibro-viscometer from Malvern. For viscosity measurements, the dispersions were analyzed without modification. The conductivity of the dispersions was calculated on a four-point probe Veeco/Miller FPP5000 using layer resistivity function.

4.2. Scaffold Preparation by Freeze-Drying. PEDOT:PSS (Heraeous PH 1000) (0.5 g) per $10 \mathrm{~mL}$ of dispersion and $0.1 \mathrm{~g}$ of DVS (Acros) per $10 \mathrm{~mL}$ of dispersion were added to each PEDOT:xanthan gum dispersion. Scaffolds were formed by freeze-drying this polymeric mixture with a LyoQuest-85 apparatus from Telstar. After freeze-drying, the scaffolds were heated up at $50{ }^{\circ} \mathrm{C}$ for $1 \mathrm{~h}$ at mild vacuum $(0.5$ bar) for evaporation of DVS in excess. Scaffolds were freezedried in cylindrical PDMS (from Dow Corning) molds of two dimensions: $8 \mathrm{~mm}$ diameter and $8 \mathrm{~mm}$ height for mechanical testing and $3 \mathrm{~mm}$ diameter and $2 \mathrm{~mm}$ height for impedance measurements and cell culture.

4.2.1. Scaffold Characterization. Scaffolds were characterized by SEM and mechanically using Instron. SEM images were taken with a Zeiss Sigma FESEM equipment. With an Instron apparatus, the compressive moduli of the dry and hydrated scaffolds (submerged $2 \mathrm{~h}$ in standard Dulbecco's PBS solution) were calculated. The Instron had a load cell of $1 \mathrm{kN}$ and the compression speed was set at $1 \mathrm{~mm} \mathrm{~min}^{-1}$. Young's modulus values were calculated from the slope of the linear part of the strain stress curve. Scaffold density was calculated according to their volume and weight. Swelling analysis of the scaffolds in cell media was calculated by measuring the wet scaffold weight at certain time intervals after removing excess surface media.

4.3. Scaffold Cell Culture. MDCK II cells were kindly provided by Frederic Luton (IPMC, Valbonne). MDCK II LifeAct cells were prepared for this study in the same manner according to the manufacturer guidelines (Ibidi, $\mathrm{GmbH}$ ) and as described previously using pCMVLifeAct-TagRFP. ${ }^{44}$ Cells were cultured in Dulbecco's modified Eagle medium low glucose supplemented with $10 \%$ fetal bovine serum, $2 \times 10^{-3}$ $\mathrm{M}$ glutamine, $50 \mathrm{U} \mathrm{mL} \mathrm{m}^{-1}$ penicillin, and $50 \mu \mathrm{g} \cdot \mathrm{mL}^{-1}$ streptomycin. To keep pressure on the fluorescent actin expression, $500 \mu \mathrm{g} \cdot \mathrm{mL}^{-1}$ geneticin was added to the media. MDCK II eGFP (gift from Frederic Luton) was cultured in the same media as MDCK II cells.

Prior to any experiment, scaffolds were dipped into ethanol $70 \%$ for $1 \mathrm{~h}$ for sterilization and then washed three times $1 \mathrm{~h}$ each with sterile water to ensure that all ethanol was removed. Scaffolds were kept in cell media for $1 \mathrm{~h}$ before being dried using sterile absorbers. Scaffolds were dipped into $2 \mathrm{~mL}$ of $6 \times$ $10^{6}$ MDCK II cells in suspension allowing cell penetration by capillarity. After cell seeding, the scaffolds were incubated for 1 night at $37{ }^{\circ} \mathrm{C}$ to allow the cells to adhere, after which $5 \mathrm{~mL}$ of fresh media was added to the well and half-changed every 2 days until scaffolds were ready.

4.3.1. Fluorescent Images. MDCK II cells were fixed in $4 \%$ paraformaldehyde for $10 \mathrm{~min}$ at room temperature. The scaffolds were washed extensively with PBS and water before being monitored under a epifluorescence/confocal microscope (AxioObserver Z1 LSM 800 ZEISS).

4.3.2. Scaffold Electrical Characterization. Scaffolds were characterized by EIS using an Autolab potentiostat in the frequency range $10^{-1}$ to $10^{5} \mathrm{~Hz}$. A commercial $\mathrm{Ag} / \mathrm{AgCl} / \mathrm{KCl}$ ( $3 \mathrm{M}$ ) electrode from Metrohm and a platinum mesh were employed as the reference and counter electrode, respectively. The cell culture medium was the electrolyte solution.

\section{ASSOCIATED CONTENT}

\section{Supporting Information}

The Supporting Information is available free of charge on the ACS Publications website at DOI: 10.1021/acsomega.8b00458.

$$
\text { Scaffold swelling ratio (PDF) }
$$

\section{AUTHOR INFORMATION}

\section{Corresponding Authors}

*E-mail: rmo37@cam.ac.uk (R.M.O.).

*E-mail: david.mecerreyes@ehu.es (D.M.).

ORCID

George G. Malliaras: 0000-0002-4582-8501

David Mecerreyes: 0000-0002-0788-7156

\section{Notes}

The authors declare no competing financial interest.

\section{ACKNOWLEDGMENTS}

The work was supported by EU through the Projects FP7PEOPLE-2012-ITN 316832-OLIMPIA and FP7-PEOPLE2013-ITN 607896-OrgBio. The authors would like to thank G. Mermans for the scaffold drawing and P. Leal for the SEM image of the dispersion. A.S.-S. is thankful for the Marie Curie IF BIKE project no. 742865. D.I. acknowledges support from 
the Marie Skłodowska-Curie Individual Fellowship (IF-EF) (Smart-Bone Project, grant agreement no. 704175).

\section{REFERENCES}

(1) Drury, J. L.; Mooney, D. J. Hydrogels for Tissue Engineering: Scaffold Design Variables and Applications. Biomaterials 2003, 24, 4337-4351.

(2) Chan, B. P.; Leong, K. W. Scaffolding in Tissue Engineering: General Approaches and Tissue-Specific Considerations. Eur. Spine J. 2008, 17, 467-479.

(3) Loh, Q. L.; Choong, C. Three-Dimensional Scaffolds for Tissue Engineering Applications: Role of Porosity and Pore Size. Tissue Eng., Part B 2013, 19, 485-502.

(4) Lawrence, B. J.; Madihally, S. V. Cell Colonization in Degradable 3D Porous Matrices. Cell Adhes. Migr. 2008, 2, 9.

(5) Bueno, V. B.; Takahashi, S. H.; Catalani, L. H.; de Torresi, S. I. C.; Petri, D. F. S. Biocompatible Xanthan/polypyrrole Scaffolds for Tissue Engineering. Mater. Sci. Eng., C 2015, 52, 121-128.

(6) Ghasemi-Mobarakeh, L.; Prabhakaran, M. P.; Morshed, M.; Nasr-Esfahani, M. H.; Baharvand, H.; Kiani, S.; Al-Deyab, S. S.; Ramakrishna, S. Application of Conductive Polymers, Scaffolds and Electrical Stimulation for Nerve Tissue Engineering. J. Tissue Eng. Regener. Med. 2011, 5, e17-e35.

(7) Wu, J. C.-C.; Ray, S.; Gizdavic-Nikolaidis, M.; Uy, B.; Swift, S.; Jin, J.; Cooney, R. P. Nanostructured Bioactive Material Based on Polycaprolactone and Polyaniline Fiber-Scaffolds. Synth. Met. 2014, $198,41-50$.

(8) Sarvari, R.; Akbari-Alanjaraghi, M.; Massoumi, B.; BeygiKhosrowshahi, Y.; Agbolaghi, S. Conductive and Biodegradable Scaffolds Based on a Five-Arm and Functionalized Star-like Polyaniline-polycaprolactone Copolymer with a D-Glucose Core. New J. Chem. 2017, 41, 6371-6384.

(9) Jaymand, M.; Sarvari, R.; Abbaszadeh, P.; Massoumi, B.; Eskandani, M.; Beygi-Khosrowshahi, Y. Development of Novel Electrically Conductive Scaffold Based on Hyperbranched Polyester and Polythiophene for Tissue Engineering Applications. J. Biomed. Mater. Res., Part A 2016, 104, 2673-2684.

(10) Hatamzadeh, M.; Najafi-Moghadam, P.; Baradar-Khoshfetrat, A.; Jaymand, M.; Massoumi, B. Novel Nanofibrous Electrically Conductive Scaffolds Based on Poly(ethylene Glycol)s-Modified Polythiophene and $\operatorname{Poly}(\varepsilon$-Caprolactone $)$ for Tissue Engineering Applications. Polymer 2016, 107, 177-190.

(11) Marzocchi, M.; Gualandi, I.; Calienni, M.; Zironi, I.; Scavetta, E.; Castellani, G.; Fraboni, B. Physical and Electrochemical Properties of PEDOT:PSS as a Tool for Controlling Cell Growth. ACS Appl. Mater. Interfaces 2015, 7, 17993-18003.

(12) Lari, A.; Sun, T.; Sultana, N. PEDOT: PSS-Containing Nanohydroxyapatite/Chitosan Conductive Bionanocomposite Scaffold: Fabrication and Evaluation. J. Nanomater. 2016, 2016, 1-12.

(13) Wang, Q. Smart Materials for Tissue Engineering; Smart Materials Series; The Royal Society of Chemistry, 2017.

(14) Balint, R.; Cassidy, N. J.; Cartmell, S. H. Conductive Polymers: Towards a Smart Biomaterial for Tissue Engineering. Acta Biomater. 2014, 10, 2341-2353.

(15) Wang, S.; Guan, S.; Xu, J.; Li, W.; Ge, D.; Sun, C.; Liu, T.; Ma, $X$. Neural Stem Cell Proliferation and Differentiation in the Conductive PEDOT-HA/Cs/Gel Scaffold for Neural Tissue Engineering. Biomater. Sci. 2017, 5, 2024-2034.

(16) Wang, S.; Sun, C.; Guan, S.; Li, W.; Xu, J.; Ge, D.; Zhuang, M.; Liu, T.; Ma, X. Chitosan/gelatin Porous Scaffolds Assembled with Conductive poly(3,4-Ethylenedioxythiophene) Nanoparticles for Neural Tissue Engineering. J. Mater. Chem. B 2017, 5, 4774-4788.

(17) Zubair, N. A.; Rahman, N. A.; Lim, H. N.; Sulaiman, Y. Production of Conductive PEDOT-Coated PVA-GO Composite Nanofibers. Nanoscale Res. Lett. 2017, 12, 113.

(18) Richardson-Burns, S. M.; Hendricks, J. L.; Martin, D. C. Electrochemical Polymerization of Conducting Polymers in Living Neural Tissue. J. Neural Eng. 2007, 4, L6-L13.
(19) Wan, A. M.-D.; Inal, S.; Williams, T.; Wang, K.; Leleux, P.; Estevez, L.; Giannelis, E. P.; Fischbach, C.; Malliaras, G. G.; Gourdon, D. 3D Conducting Polymer Platforms for Electrical Control of Protein Conformation and Cellular Functions. J. Mater. Chem. B 2015, 3, 5040-5048.

(20) Inal, S.; Hama, A.; Ferro, M.; Pitsalidis, C.; Oziat, J.; Iandolo, D.; Pappa, A.-M.; Hadida, M.; Huerta, M.; Marchat, D.; et al. Conducting Polymer Scaffolds for Hosting and Monitoring 3D Cell Culture. Adv. Biosyst. 2017, 1, 1700052.

(21) Ma, Z.; Mao, Z.; Gao, C. Surface Modification and Property Analysis of Biomedical Polymers Used for Tissue Engineering. Colloids Surf., B 2007, 60, 137-157.

(22) Aamodt, J. M.; Grainger, D. W. Extracellular Matrix-Based Biomaterial Scaffolds and the Host Response. Biomaterials 2016, 86, $68-82$.

(23) Hinderer, S.; Layland, S. L.; Schenke-Layland, K. ECM and ECM-like Materials - Biomaterials for Applications in Regenerative Medicine and Cancer Therapy. Adv. Drug Deliv. Rev. 2016, 97, 260269.

(24) Petri, D. F. S. Xanthan Gum: A Versatile Biopolymer for Biomedical and Technological Applications. J. Appl. Polym. Sci. 2015, 132, 42035.

(25) Kumar, A.; Rao, K. M.; Han, S. S. Application of Xanthan Gum as Polysaccharide in Tissue Engineering: A Review. Carbohydr. Polym. 2018, 180, 128-144.

(26) Bueno, V. B.; Bentini, R.; Catalani, L. H.; Barbosa, L. R. S.; Petri, D. F. S. Synthesis and Characterization of Xanthanhydroxyapatite Nanocomposites for Cellular Uptake. Mater. Sci. Eng., C 2014, 37, 195-203.

(27) Harman, D. G.; Gorkin, R.; Stevens, L.; Thompson, B.; Wagner, K.; Weng, B.; Chung, J. H. Y.; in het Panhuis, M.; Wallace, G. G. Poly(3,4-Ethylenedioxythiophene):dextran Sulfate (PEDOT: DS) - A Highly Processable Conductive Organic Biopolymer. Acta Biomater. 2015, 14, 33-42.

(28) Hai, W.; Goda, T.; Takeuchi, H.; Yamaoka, S.; Horiguchi, Y.; Matsumoto, A.; Miyahara, Y. Specific Recognition of Human Influenza Virus with PEDOT Bearing Sialic Acid-Terminated Trisaccharides. ACS Appl. Mater. Interfaces 2017, 9, 14162-14170.

(29) Hofmann, A. I.; Katsigiannopoulos, D.; Mumtaz, M.; Petsagkourakis, I.; Pecastaings, G.; Fleury, G.; Schatz, C.; Pavlopoulou, E.; Brochon, C.; Hadziioannou, G.; et al. How To Choose Polyelectrolytes for Aqueous Dispersions of Conducting PEDOT Complexes. Macromolecules 2017, 50, 1959-1969.

(30) Wang, W.; Cui, M.; Song, Z.; Luo, X. An Antifouling Electrochemical Immunosensor for Carcinoembryonic Antigen Based on Hyaluronic Acid Doped Conducting Polymer PEDOT. RSC Adv. 2016, 6, 88411-88416.

(31) Mantione, D.; del Agua, I.; Schaafsma, W.; Diez-Garcia, J.; Castro, B.; Sardon, H.; Mecerreyes, D. Poly(3,4-ethylenedioxythiophene):GlycosAminoGlycan Aqueous Dispersions: Toward Electrically Conductive Bioactive Materials for Neural Interfaces. Macromol. Biosci. 2016, 16, 1227-1238.

(32) del Agua, I.; Mantione, D.; Casado, N.; Sanchez-Sanchez, A.; Malliaras, G. G.; Mecerreyes, D. Conducting Polymer Iongels Based on PEDOT and Guar Gum. ACS Macro Lett. 2017, 6, 473-478.

(33) Massonnet, N.; Carella, A.; Jaudouin, O.; Rannou, P.; Laval, G.; Celle, C.; Simonato, J.-P. Improvement of the Seebeck Coefficient of PEDOT: PSS by Chemical Reduction Combined with a Novel Method for Its Transfer Using Free-Standing Thin Films. J. Mater. Chem. C 2014, 2, 1278-1283.

(34) Cruz-Cruz, I.; Reyes-Reyes, M.; Rosales-Gallegos, I. A.; Gorbatchev, A. Y.; Flores-Camacho, J. M.; López-Sandoval, R. Visible Luminescence of Dedoped DBU-Treated PEDOT: PSS Films. J. Phys. Chem. C 2015, 119, 19305-19311.

(35) Mantione, D.; del Agua, I.; Schaafsma, W.; ElMahmoudy, M.; Uguz, I.; Sanchez-Sanchez, A.; Sardon, H.; Castro, B.; Malliaras, G. G.; Mecerreyes, D. Low-Temperature Cross-Linking of PEDOT: PSS Films Using Divinylsulfone. ACS Appl. Mater. Interfaces 2017, 9, 18254-18262. 
(36) del Agua, I.; Mantione, D.; Ismailov, U.; Sanchez-Sanchez, A.; Aramburu, N.; Malliaras, G. G.; Mecerreyes, D.; Ismailova, E. DVSCrosslinked PEDOT: PSS Free-Standing and Textile Electrodes toward Wearable Health Monitoring. Adv. Mater. Technol. 2018, 1700322.

(37) O’Brien, F. J. Biomaterials \& Scaffolds for Tissue Engineering. Mater. Today 2011, 14, 88-95.

(38) Murphy, C. M.; Haugh, M. G.; O’Brien, F. J. The Effect of Mean Pore Size on Cell Attachment, Proliferation and Migration in Collagen-glycosaminoglycan Scaffolds for Bone Tissue Engineering. Biomaterials 2010, 31, 461-466.

(39) Murphy, C. M.; O’Brien, F. J.; Little, D. G.; Schindeler, A. CellScaffold Interactions in the Bone Tissue Engineering Triad. Eur. Cells Mater. 2013, 26, 120-132.

(40) Guex, A. G.; Puetzer, J. L.; Armgarth, A.; Littmann, E.; Stavrinidou, E.; Giannelis, E. P.; Malliaras, G. G.; Stevens, M. M. Highly Porous Scaffolds of PEDOT: PSS for Bone Tissue Engineering. Acta Biomater. 2017, 62, 91-101.

(41) Engler, A. J.; Sen, S.; Sweeney, H. L.; Discher, D. E. Matrix Elasticity Directs Stem Cell Lineage Specification. Cell 2006, 126, 677-689.

(42) Karimullah, A.; Cumming, R. S.; Riehle, M.; Gadegaard, N. Development of a Conducting Polymer Cell Impedance Sensor. Sens. Actuators, B 2013, 176, 667-674.

(43) Atanasov, V.; Knorr, N.; Duran, R. S.; Ingebrandt, S.; Offenhäusser, A.; Knoll, W.; Köper, I. Membrane on a Chip: A Functional Tethered Lipid Bilayer Membrane on Silicon Oxide Surfaces. Biophys. J. 2005, 89, 1780-1788.

(44) Ramuz, M.; Hama, A.; Rivnay, J.; Leleux, P.; Owens, R. M. Monitoring of Cell Layer Coverage and Differentiation with the Organic Electrochemical Transistor. J. Mater. Chem. B 2015, 3, 59715977. 